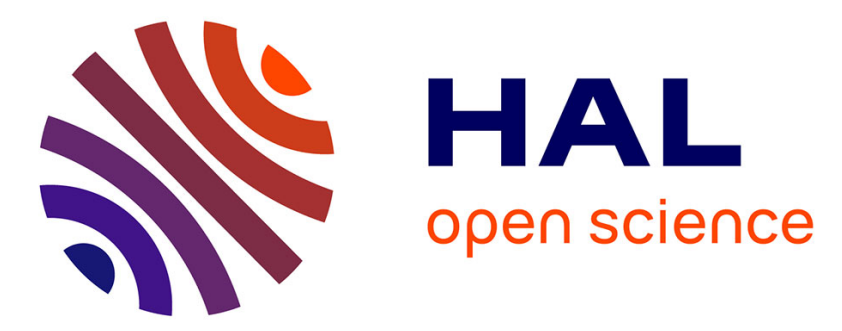

\title{
An automatic and simplified approach to muscle path modelling
}

Claire Livet, Théo Rouvier, Georges Dumont, Charles Pontonnier

\section{To cite this version:}

Claire Livet, Théo Rouvier, Georges Dumont, Charles Pontonnier. An automatic and simplified approach to muscle path modelling. Journal of Biomechanical Engineering, 2021, 144 (1), pp.1-9. 10.1115/1.4051870 . hal-03279707

\section{HAL Id: hal-03279707 https://inria.hal.science/hal-03279707}

Submitted on 8 Jul 2021

HAL is a multi-disciplinary open access archive for the deposit and dissemination of scientific research documents, whether they are published or not. The documents may come from teaching and research institutions in France or abroad, or from public or private research centers.
L'archive ouverte pluridisciplinaire HAL, est destinée au dépôt et à la diffusion de documents scientifiques de niveau recherche, publiés ou non, émanant des établissements d'enseignement et de recherche français ou étrangers, des laboratoires publics ou privés. 


\title{
An automatic and simplified approach to muscle path modelling
}

\author{
Claire Livet \\ Univ Rennes, Inria, CNRS, IRISA \\ France \\ Email: claire.livet@irisa.fr \\ Théo Rouvier \\ Institut de Biomécanique Humaine Georges Charpak \\ Arts et Métiers Institute of Technology \\ France \\ Email: theo.rouvier@ensam.eu \\ Georges Dumont \\ Univ Rennes, Inria, CNRS, IRISA \\ France \\ Email: georges.dumont@irisa.fr \\ Charles Pontonnier \\ Univ Rennes, Inria, CNRS, IRISA \\ France \\ Email: charles.pontonnier@irisa.fr
}

The current paper aims at proposing an automatic method to design and adjust simplified muscle paths of a musculoskeletal model. These muscle paths are composed of straight-lines described by a limited set of fixed active via points and an optimization routine is developed to place these via points on the model in order to fit moment arms and musculotendon lengths input data. The method has been applied to a forearm musculoskeletal model extracted from the literature, using theoretical input data as an example. Results showed that for $75 \%$ of the muscle set, the relative root mean square error between literature theoretical data and the results from optimized muscle path was under $29.23 \%$ for moment arms and of $1.09 \%$ for musculotendon lengths. These results confirm the ability of the method to automatically generate computationally efficient muscle paths for musculoskeletal simulations. Using only via points lowers computational expense compared to paths exhibiting wrapping objects. A proper balance between computational time and anatomical realism should be found to help those models being interpreted by practitioners.

Index terms - Moment arm, Musculotendon length, Musculoskeletal model, Forearm

\section{Nomenclature}

$n_{\tilde{q}} \in \mathbb{R} \quad$ number of degrees of freedom actuated by a muscle $A_{i} \subset \mathbb{R}$ range of motion of the $i$ th degree of freedom $\tilde{q}_{i} \in A_{i} \quad i$ th degree of freedom actuated by a muscle

$\tilde{\mathbf{q}} \in \prod_{i=1}^{n_{\tilde{q}}} A_{i} \quad$ vector of degrees of freedom actuated by a muscle

$n_{s} \in \mathbb{R} \quad$ number of segments on the muscle path. $n_{s}$ may be greater than $n_{\tilde{q}}$

$n_{m} \in \mathbb{R} \quad$ number of joint with single degree of freedom

$p(j) \in \mathbb{N}$ predecessor segment of joint $j$

$s(j) \in \mathbb{N} \quad$ successor segment of joint $j$

$C^{k} \in \mathbb{R}^{3} \quad$ cylinder representing the shape of the segment $k$

$\mathbf{X}_{j}^{k} \in C^{k} \quad$ via point vector position belonging to segment $k$ acting on joint $j$, with $k \in p(j), s(j)$.

$\mathbf{X} \in \prod_{k=1}^{n_{s}} C^{k} \quad$ vector of all via points

$L_{\text {input }}(\tilde{\mathbf{q}}) \in \mathbb{R}$ input musculotendon path length

$L(\tilde{\mathbf{q}}) \in \mathbb{R}$ model musculotendon path length

$R_{\text {input }}^{\tilde{q}_{i}}(\tilde{\mathbf{q}}) \in \mathbb{R}$ input moment arm associated to $\tilde{q}_{i}$

$R^{\tilde{q}_{i}}(\tilde{\mathbf{q}}, \mathbf{X}) \in \mathbb{R} \quad$ model moment arm associated to $\tilde{q}_{i}$

$\mathbf{O}_{\text {input }} \in \mathbb{R}^{3}$ input origin point

$\mathbf{O} \in \mathbb{R}^{3} \quad$ model origin point

$\mathbf{I}_{\text {input }} \in \mathbb{R}^{3}$ input insertion point 
$\mathbf{I} \in \mathbb{R}^{3} \quad$ model insertion point

$\lambda \in \mathbb{R}$ homothety factor

$\theta \in \mathbb{R}^{n_{m}} \quad$ angles of rotation

\section{Introduction}

Muscle moment arms and musculotendon lengths play an important role in the muscle forces sharing problem in musculoskeletal simulation. Indeed, the potential contribution of a muscle to a joint motion depends on its geometrical implementation - its geometrical path, with regard to the joint. This path will directly define the capacity of the muscle to actuate a joint via the definition of its moment arm and its inner length [1]. It is therefore important to properly implement this path to provide a realistic muscle actuation to a musculoskeletal model.

Imaging techniques [2-4] and anatomical landmarks [57] can be used to estimate and model muscle paths. Such data can be very helpful to model objects to lead the muscle path. Via points (VP) can be used in straight-lines or centroidlines methods [8]. Obstacles, like spheres, cylinders, ellipsoids (generally denominated wrapping objects) $[3,5,6]$, can simulate wrapping around bone prominences for obstacleset methods. These via points and wrapping objects affect moment arms and musculotendon lengths as well. Wrapping objects parameters are sometimes adjusted manually $[6,7,9]$, however this work is long and tedious [10]. The creation of these models requires technical $[11,12]$ and experimental expertise in data fitting and muscle modeling, while increasing the cost of computation during simulations [12] since wrapping trajectories must be computed at each time step.

Several methods using experimental moment arms data to automatically define muscle path parameters have been proposed $[10,12,13]$. The paths resulting from these methods save fitting time but can contain many wrapping objects. Moreover, the methods developed by $[10,13]$ require to define a priori the obstacles to be placed for each muscle. Lastly, the geometric parameters of multiple wrapping objects prevent simple scaling from a generic model to a subject-specific model [12] since scaling rules (homothetic [14], radial [15]) may not be relevant for such objects.

These studies show that there is a lack of methods able to: a) automatize muscle path modelling from experimental data; b) reduce the computation time for muscle lengths and moment arms during moment arm computation for muscle forces estimation in musculoskeletal simulation; c) facilitate the geometrical scaling of the musculoskeletal model.

The current paper aims at proposing an automatic method to model and adjust simplified muscle paths of a musculoskeletal model. The muscle paths are composed of a limited set of fixed active via points and optimization routines are developed to fit moment arms and musculotendon lengths input data. The method can be used to describe a generic model as well as for a subject-specific model. As an example, the method has been applied to a forearm musculoskeletal model issued from the literature [16], using theoretical input data [17].

The aim of the following method is to automatically set via points to obtain moment arms and musculotendon lengths values as close as possible to moment arms and musculotendon lengths inputs. The needed muscle data inputs are: moment arms data, musculotendon lengths data, muscle origin and insertion points. Input data can be issued from experiments or from literature models.

Fitting procedures based on an optimization scheme are performed: a first one minimizing the difference between the moment arms issued from the input data and those computed by the model, a second one between lengths issued from the input data and those computed by the model, with spatial positions of via points as decision variables. The first procedure also takes into account spatial constraints, so that the final muscle is contained within a consistent body volume, which represents human flesh volume where muscles lie. Since muscles actuate joints, the method relies on an osteoarticular model to compute moment arms and musculotendon lengths. The same osteoarticular model and body volumes can be used for all muscles.

The outline of the paper is as follow: firstly, we describe the muscle path model and the method we use to tune it from generic input data. An overview of this method is illustrated in Figure 1, describing inputs and outputs as well as successive optimization stages. The application case on an upper limb model for a middle size subject is then developed and results are presented and discussed. The computational and anatomical realism of the results are discussed with regard to the aforementioned objectives.

\section{Materials and Methods}

\subsection{Muscle path model definition Moment arm definition}

Among all the possible definitions of the muscle moment arm described in [18], the definition given in [19] is chosen, where $R^{\tilde{q}_{i}}$ is the muscle moment arm, $L(\tilde{\mathbf{q}})$ the musculotendon path length and $\tilde{q}_{i}$ the functional degrees of freedom:

$$
R^{\tilde{q}_{i}}(\tilde{\mathbf{q}})=\frac{\partial L(\tilde{\mathbf{q}})}{\partial \tilde{q}_{i}}
$$

Indeed, they are the coefficients representing the contribution of muscle efforts in the equations of dynamics. This definition is relevant to properly actuate functional degrees of freedom of the human body.

\section{Muscle types}

In this method, muscles are divided in two categories because the problem to solve in moment arm optimization depends on these categories. Muscles categories are:

- Type 1: the muscle actuates at least a joint with only one degree of freedom (DOF)

- Type 2: the muscle actuates only joints with more than one DOF 


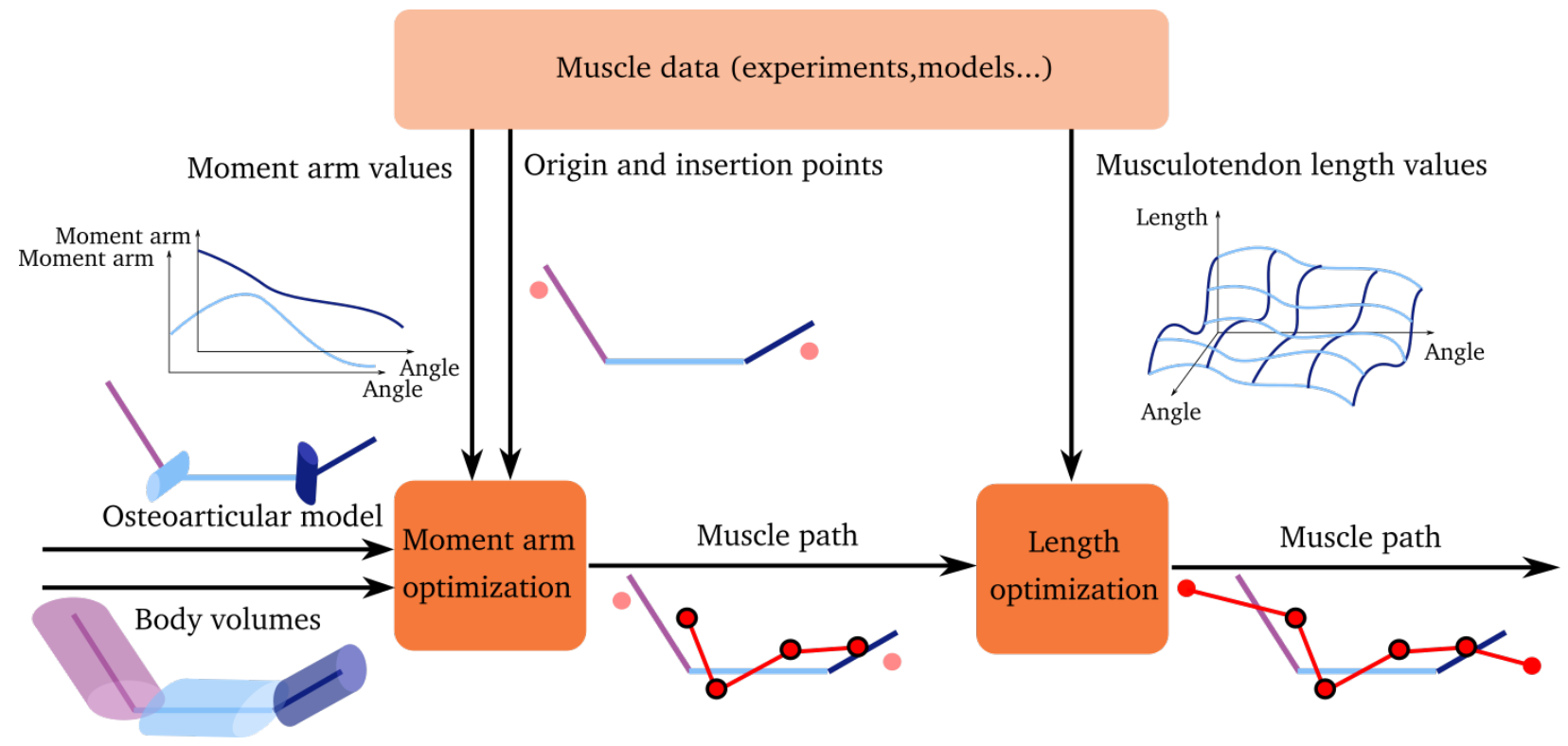

Figure 1: Overview of the muscle path design method

\section{Geometrical path}

Every muscle path is composed of straight lines systematically built using only muscle origin, muscle insertion, and fixed active via points. Muscles with multiple heads are artificially divided into independent muscles.

We propose to use only two via points for each joint, i.e. one via point per segment articulated by the joint. Examples of muscles paths illustrate this in Figure 2, where the number of via points (black circles) changes with the number of segments. Using two via points per joint enables us to compensate possible misplacement of origin and insertion points.

For a muscle actuating a single joint, one via point on each side of the joint guarantees the independence of moment arms from origin and insertion points. Indeed, the distance between pairs of via points and origin or insertion points remains constant with the variation of joint parameters, so origin and insertion points have no impact on moment arm values.

Adding a third via point has no impact on the moment arm on the joint, because two of them would belong to the same segment. Their distance would remain constant while the joint angles are varying, so the moment arm does not change.

Using only one via point enables the distance between the via point and the origin or insertion points to vary with the joint parameters. Therefore, the position of origin or insertion points would have an impact on moment arm, which is against the hypothesis of the independence of moment arm from origin and insertion points.

For a muscle actuating two serial joints, its path is also composed of two via points per joint. With this rule, two via points belong to the same segment between the two joints. The first pair of via points impacts only on the first joint moment arm, independently of the second pair of via points, and inversely.

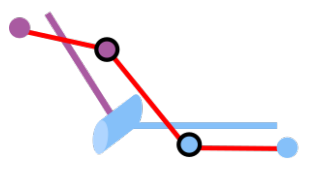

Single joint and type 1 muscle

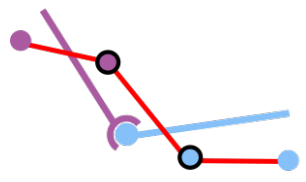

Single joint and type 2 muscle

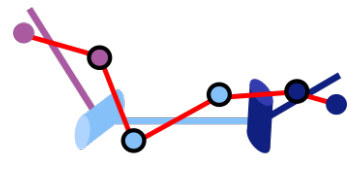

Multiple joints and type 1 muscle

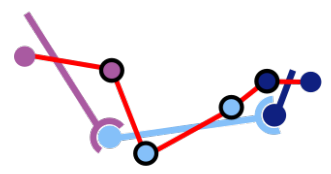

Multiple joints and type 2 muscle
Figure 2: Examples of geometrical muscle paths depending on the number of segments

\section{Spatial boundaries}

Cylinders define consistent body volumes by delimiting spatial via point positions. The muscle path is entirely contained between origin and insertion points, with no possible u-turn, as it is physiologically observed. Cylinders are delimited in length by origin and insertion points. The origin frame of each segment, taken from the osteoarticular model, delimits the length too. Points delimiting cylinders length are called reference points. The cylinders radii represent the maximum distance from the bone to the skin.

Two examples of cylinders are shown in Figures 3 and 4. Figure 3 illustrates a case of a muscle actuating three consecutive segments. For instance, humerus, radius and hand segments can be represented by that serial chain. Figure 4 represents a case when a muscle actuates two non-consecutive segments who share the same predecessor, like the ulna and radius share the humerus as predecessor. 


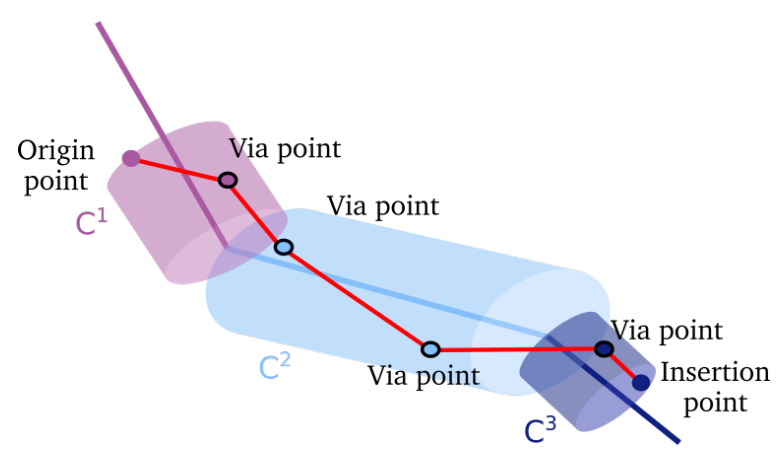

Figure 3: Cylinders for a three segments open-loop chain. Cylinders are delimited in length by reference points in full color. Via points (black circles) must stay in the cylinder of the same color.

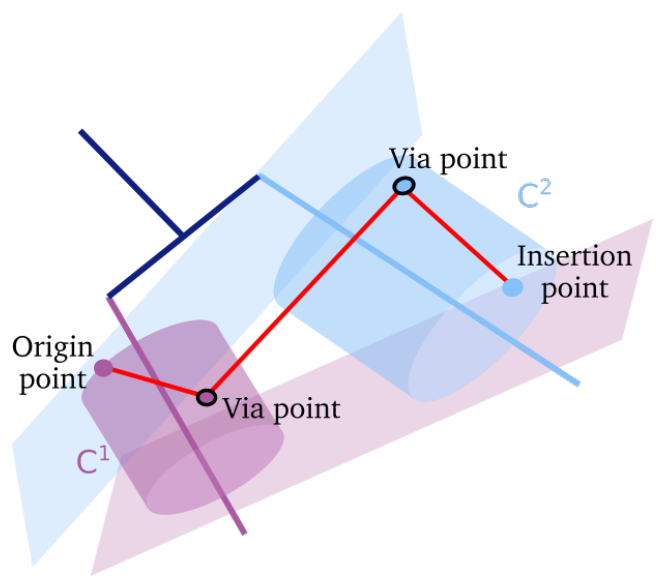

Figure 4: Cylinders for two segments with the same predecessor segment. The beginning and end faces of the cylinders are the planes perpendicular to the main axis of the segment containing the origin or insertion of the muscle.

\subsection{Fitting moment arms}

\subsubsection{Type 1 muscles}

A first approach to automatically place via points is to directly fit the model moment arm to an input moment arm. However, as it can be seen in Figure 5, there are an infinite number of via point positions giving the same moment arm for a type 1 muscle.

In Figure 5, two different positions of via points are considered. Via points A1 and B1 belong to the segment 1, while via points $\mathrm{A} 2$ and $\mathrm{B} 2$ belong to the segment 2 . The $\mathrm{B}$ triangle is a rotation of the $A$ triangle by the angle $\theta$. When the segment 2 rotates around the joint, A2 and B2 will move of the same angle, and the two triangles will still be the same triangle rotated of angle $\theta$. We conclude that there is an infinity of via points positions which lead to the same moment arm, and those positions can all be deduced from one given position and an angle $\theta$.

Therefore, for a type 1 muscle, a set of via point positions should be obtained by choosing the shortest musculotendon length $L(\tilde{\mathbf{q}}, \mathbf{X})$ under body volume constraints and moment arm fitting. In fact, musculotendon length is the

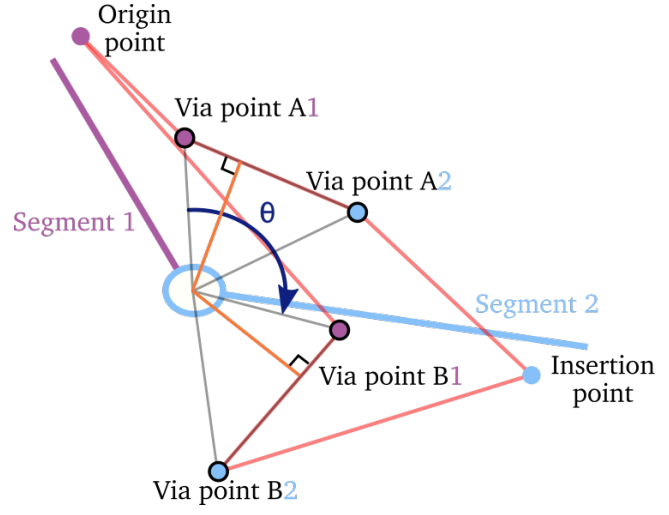

Figure 5: Example of positions of via points that lead to the same moment arm. The $B$ triangle is a rotation of the A triangle by the angle $\theta$.

shortest path from origin to insertion [18] while circumventing obstacles.

For each type 1 muscle, the equation to solve is:

$$
\begin{gathered}
\min _{\mathbf{X}} L(\tilde{\mathbf{q}}, \mathbf{X}) \\
\text { subject to }\left\{\begin{array}{l}
\forall j \in \llbracket 1, n_{s} \rrbracket, \forall k \in\{p(j), s(j)\}: \mathbf{X}_{j}^{k} \in C^{k} \\
\mathbf{X} \in \arg \min \sum_{i=1}^{n_{\tilde{q}}} \frac{\left\|R_{\text {input }}^{\tilde{q}_{i}}(\tilde{\mathbf{q}})-R^{\tilde{q}_{i}}(\tilde{\mathbf{q}}, \mathbf{X})\right\|^{2}}{\left\|R_{\text {input }}^{\tilde{q}_{i}}(\tilde{\mathbf{q}})\right\|^{2}}
\end{array}\right.
\end{gathered}
$$

(2) is an optimization to find the shortest musculotendon path that leads to the closest moment arm to the input data, under a spatial physiological constraint.

As shown in Figure 5, one only needs to find one configuration of $\mathbf{X}$ that minimizes the distance between the input moment arm and the model moment arm, and then use a rotation of $\theta$ around the single DOF joints as a new variable.

Once an initial vector position ${ }^{0} \mathbf{X}$ is found respecting (2) constraints, the problem to solve can be written as:

$$
\min _{\theta} L(\tilde{\mathbf{q}}, \theta)
$$

subject to $\forall j \in\left[\left[1, n_{s}\right]\right], \forall k \in\{p(j), s(j)\}: \mathbf{X}_{j}^{k}\left(\theta,{ }^{0} \mathbf{X}\right) \in C^{k}$

\subsubsection{Type 2 muscles}

For a type 2 muscle, fitting model moment arm and input moment arm can be done directly. With more than one degree of freedom, there is not an infinity of via point positions leading to the same moment arm.

For each type 2 muscle, the equation to solve is:

$$
\min _{\mathbf{X}} \sum_{i=1}^{n_{\tilde{q}}} \frac{\left\|R_{\text {input }}^{\tilde{q}_{i}}(\tilde{\mathbf{q}})-R^{\tilde{q}_{i}}(\tilde{\mathbf{q}}, \mathbf{X})\right\|^{2}}{\left\|R_{\text {input }}(\tilde{\mathbf{q}})\right\|^{2}}
$$

subject to $\forall j \in\left[\left[1, n_{s}\right]\right], \forall k \in\{p(j), s(j)\}: \mathbf{X}_{j}^{k} \in C^{k}$

(4) is the generalization of (2) constraints for moment arms. The moment arm error is normalized to give the same 
weight to each moment arm of the path, despite their variation range.

\subsection{Fitting musculotendon length}

Since the moment arm of the new muscle path is the derivative of musculotendon length, a constant still remains between model length and input length. The final step is to decrease this constant. The origin and insertion points are linearly scaled to perform this, since they don't influence the moment arm.

The scaling principle is represented in Figure 6. Via points are represented with black circles while origin and insertion points are represented by full color disks. Input origin and insertion points have a lighter color than the new origin and insertion points. Those new points must belong to the half-line starting from the nearest via point and passing by the input origin and insertion points.

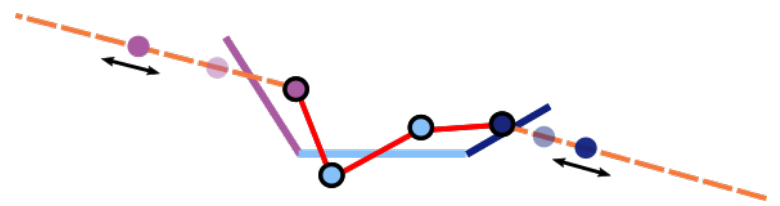

Figure 6: Adjusting origin and insertion point to fit input length

Musculotendon length fitting is described in (5): the goal is to find new origin and insertions points to match model- and input length, while positions of new points are ruled by an homothetic transformation.

$$
\begin{gathered}
\min _{\lambda}\left\|L_{\text {input }}(\tilde{\mathbf{q}})-L(\tilde{\mathbf{q}}, \lambda)\right\|^{2} \\
\text { subject to }\left\{\begin{array}{l}
\mathbf{O} \mathbf{X}_{1}^{p(1)}=\lambda \mathbf{O}_{\text {input }} \mathbf{X}_{1}^{p(1)} \\
\mathbf{I} \mathbf{X}_{n_{s}}^{p\left(n_{s}\right)}=\lambda \mathbf{I}_{\text {input }} \mathbf{X}_{n_{s}}^{p\left(n_{s}\right)}
\end{array}\right.
\end{gathered}
$$

\subsection{Performance criteria}

For each degree of freedom $\tilde{q}_{i}$ actuated by the considered muscle, root mean square error between the input data moment arm and the model is computed, in absolute (RMSE) and relative (rRMSE) terms. rRMSE is obtained by dividing the RMSE by the RMS value of the input data moment arm. This gives an estimate of the solution quality resulting from the method. The balance between two angles for a type 2 muscle is checked by the rRMSE.

Correlation and rRMSE value for musculotendon lengths comparisons are considered as performance criteria. Correlation shows the global impact of moment arm on musculotendon length.

\subsection{Use case}

To evaluate its performance, the method has been applied to a forearm model. The use case is a comparison between reference polynomial values modeling muscles created with an obstacle-set method and data coming from muscle paths generated with the method described above. Input data for this use case are:

- Osteoarticular model: closed-loop forearm model proposed by [16];

- Body volumes: complete description is given in Table 1;

- Origin and insertions points: taken from the musculoskeletal upper limb model proposed by [7];

- Moment arm input data: [17] provides polynomial coefficients to model moment arm values from [7] muscles;

- Musculotendon length input data: [17] provides polynomial coefficients to model musculotendon length values from [7] muscles.

The model proposed in [16] was linearly scaled to obtain an osteoarticular model $170 \mathrm{~cm}$ tall, similarly to the model described in [7].

The input moment arm and musculotendon length proposed in [17] interpolate muscle moment arms and musculotendon length described in [7] as polynomial functions of degrees of freedom. These polynomials were used to generate both moment arms and musculotendon lengths input data.

Anatomical segments are described in Table 1. The range of motion for each degree of freedom was taken from [17]:

- Elbow flexion extension (EFE): from $0^{\circ}$ to $100^{\circ}$

- Elbow pronation supination (PS): from $-45^{\circ}$ (supination) to $45^{\circ}$ (pronation)

- Wrist flexion extension (WFE): from $-45^{\circ}$ to $45^{\circ}$

- Wrist deviation (WDV): from $-10^{\circ}$ (radial abduction) to $25^{\circ}$ (ulnar adduction)

The axes relative to the aforementioned degrees of freedom follow the ISB recommendations [20].

Table 1: Description of segments of interest for a man (170cm tall, same as [7]'s model) with a weight of $70 \mathrm{~kg}$ (standard body mass index). Mass values were adapted from [21], density values were taken from [22] and length values were adapted from [16]. The cylinder radius was deduced from mass, density and length data.

\begin{tabular}{|l|cccc|}
\hline Segment & $\begin{array}{c}\text { Mass } \\
(\mathrm{kg})\end{array}$ & $\begin{array}{c}\text { Density } \\
\left(\mathrm{kg.L} \mathrm{L}^{-1}\right)\end{array}$ & $\begin{array}{c}\text { Length } \\
(\mathrm{m})\end{array}$ & $\begin{array}{c}\text { Cylinder } \\
\text { radius }(\mathrm{m})\end{array}$ \\
\hline Humerus & 1.67 & 1.07 & 0.32 & 0.040 \\
Radius & 0.47 & 1.13 & 0.25 & 0.023 \\
Ulna & 0.72 & 1.13 & 0.023 & 0.029 \\
Hand & 0.42 & 1.16 & 0.013 & 0.030 \\
\hline
\end{tabular}

The method was applied on muscles listed in Table 2. A comparison of muscle paths from the original model [7] 
and the proposed model is also given in Table 2, because a goal of the proposed method was to simplify muscle path description. For instance, the type 1 muscle Anconeus was modeled by only one obstacle in the original model while the proposed method used two via points instead. The type 2 muscle Pronator Teres was modeled by one obstacle and three via points in the original model, while the proposed method simplified the path with only two via points.

Table 2: Comparison of needed data to describe muscles path: figures in obstacles column and via points (VP) column correspond to the number of obstacles and the number of via points, respectively, used to describe a muscle path

\begin{tabular}{|c|c|c|c|}
\hline \multirow[t]{2}{*}{ Muscles } & \multicolumn{2}{|c|}{ Model from [7] } & \multirow{2}{*}{$\begin{array}{c}\text { Model } \\
\text { VP }\end{array}$} \\
\hline & Obstacles & VP & \\
\hline \multicolumn{4}{|l|}{ Type 1 muscles } \\
\hline Anconeus (ANC) & 1 & 0 & 2 \\
\hline Brachialis (BRA) & 0 & 2 & 2 \\
\hline Pronator Quadratus (PQ) & 2 & 0 & 2 \\
\hline Supinator Brevis (SUP) & 1 & 1 & 2 \\
\hline Triceps Medial (TRImed) & 1 & 3 & 2 \\
\hline Triceps Lateral (TRIlat) & 1 & 3 & 2 \\
\hline \multicolumn{4}{|l|}{ Type 2 muscles } \\
\hline Brachioradialis (BRD) & 1 & 1 & 2 \\
\hline $\begin{array}{l}\text { Extensor Carpi } \\
\text { Radialis Longus (ECRL) }\end{array}$ & 2 & 2 & 4 \\
\hline $\begin{array}{l}\text { Extensor Carpi } \\
\text { Radialis Brevis (ECRB) }\end{array}$ & 1 & 2 & 4 \\
\hline $\begin{array}{l}\text { Extensor Carpi } \\
\text { Ulnaris (ECU) }\end{array}$ & 1 & 4 & 4 \\
\hline $\begin{array}{l}\text { Flexor Carpi } \\
\text { Radialis (FCR) }\end{array}$ & 2 & 1 & 4 \\
\hline $\begin{array}{l}\text { Flexor Carpi } \\
\text { Ulnaris (FCU) }\end{array}$ & 2 & 2 & 4 \\
\hline Palmaris Longus (PL) & 2 & 2 & 4 \\
\hline Pronator Teres (PT) & 1 & 3 & 2 \\
\hline
\end{tabular}

The method has been implemented in CusToM [23], an open-source MATLAB ${ }^{\circledR}$ toolbox.

The moment arm optimization (4) was computed using the Global Search solver, with an interior-point method. When the reference points - origin and insertion points issued from the input data - respected the constraints of lying inside the defined cylinders, they were used as initial guesses. Otherwise, initial points which respect the cylinder constraints were chosen randomly.

The other optimization problems (3) and (5) were solved with a sequential quadratic programming algorithm, with a null initial guess.

\section{Results and Discussion \\ 3.1 Overall results}

Complete path descriptions for each muscle are reported in supplementary materials. RMSE and rRMSE for moment arms and rRMSE for musculotendon lengths are reported in supplementary materials. Examples of results are shown for moment arm for Pronator Quadratus and Palmaris Longus muscles in Figures 9 and 10. As the Pronator Quadratus muscle is a type 1 muscle, its moment arm and musculotendon length values are represented as functions of only PS angle. The Palmaris Longus muscle is a type 2 muscle. EFE and PS moment arm are independant from WDV and WFE angles, and inversely.

A synthesis of the results is given in Table 3. The median of moment arm rRMSEs was of $13.27 \%$. This result is acceptable in regards to the discrepancy in moment arms values reported in the literature. For Brachioradialis, Extensor Carpi Radialis Longus, Brachialis, Pronator Teres and Triceps muscles, [24] reports a maximum inter-specimen variability of $18 \%$ (achieved for Pronator Teres). For wrist muscles (Flexor Carpi Radialis, Flexor Carpi Ulnaris, Extensor Carpi Radialis Longus, Extensor Carpi Radialis Brevis and Extensor Carpi Ulnaris muscles), [4] reported maximum inter-specimen variability of $45.5 \%$ (achieved for Extensor Carpi Ulnaris). So, the method is relevant for $75 \%$ of the muscles (Table 3), regarding the moment arms. Some initial complex muscle models, such as the Flexor Carpi Radialis muscle, can be accurately represented using only two via points per joint. For all muscles, the original number of obstacles had no significant impact on the results.

Correlation between lengths are shown in Figure 7. The minimal correlation was 0.8435 , reported for the Extensor Carpi Ulnaris muscle. Figure 7 shows that type 1, Brachioradialis and Pronator Teres muscles were all above 0.999 of correlation. All these muscles have only two via points on their path (Table 2). They were also among those with the lowest musculotendon length rRMSE. It was deduced that for muscles actuating only one joint, a muscle path composed of origin, insertion, and two via points was enough to accurately estimate their moment arms and inner lengths.

Musculotendon lengths RMSEs were below $11.18 \%$. A comparison of musculotendon lengths before and after length optimization is given in Figure 8. Extensor Carpi Radialis Brevis muscle can be considered as an outlier, regarding the fact that all other musculotendon length rRMSEs were below $2.93 \%$. [25] reported a maximum of 53\% error difference between their model and experimental data for Extensor Carpi Radialis Brevis muscle. Compared to [25], musculotendon lengths resulting from our model were acceptable. 
Table 3: Synthesized results for all muscles. Minimum, first quantile, median, third quantile and maximum values are given for moment arm rRMSE of every muscle and for each actuated joint angle. Statistical data are also given for musculotendon length correlation and rRMSE.

\begin{tabular}{|l|c|c|c|c|c|}
\hline Criterion & Minimum & First quantile & Median & Third quantile & Maximum \\
\hline Moment arm rRMSE (\%) \\
\hline EFE axis & 2.86 & 6.41 & 7.62 & 12.07 & 34.80 \\
\hline PS axis & 0.13 & 5.20 & 8.88 & 19.22 & 57.25 \\
\hline WDV axis & 13.49 & 20.85 & 32.57 & 38.36 & 41.95 \\
\hline WFE axis & 14.43 & 18.70 & 23.57 & 44.67 & 51.75 \\
\hline All axis & 0.13 & 6.59 & 13.27 & 29.23 & 57.25 \\
\hline Musculotendon length & & & \\
\hline Correlation & 0.8435 & 0.9928 & 0.9992 & 0.9998 & 1 \\
\hline rRMSE (\%) & 0.00 & 0.20 & 0.42 & 1.09 & 11.18 \\
\hline
\end{tabular}

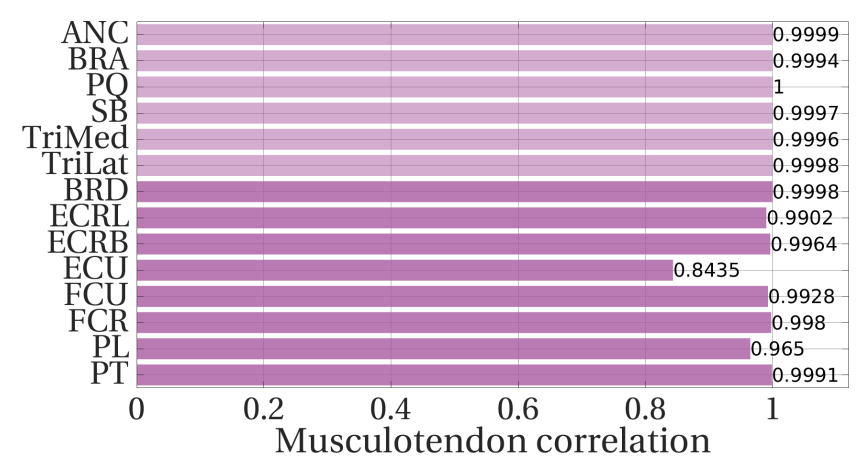

Figure 7: Correlation between musculotendon length input and model. Type 1 muscles, respectively type 2 muscles, are indicated with transparent bars, respectively opaque bars.

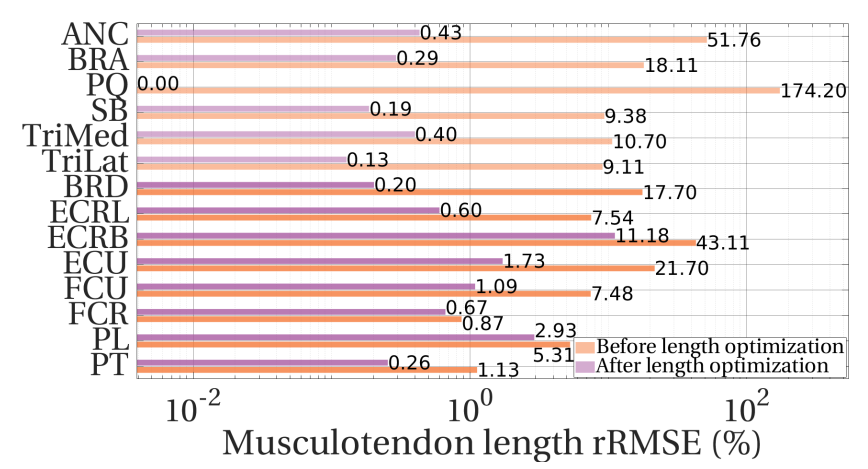

Figure 8: Musculotendon length rRMSE between musculotendon length input and model, before and after length optimization. Type 1 muscles, respectively type 2 muscles, are indicated with transparent bars, respectively opaque bars.

The relevance of the length optimization could be questionned for Supinator Brevis, Triceps Lateral, Extensor Carpi Radialis Longus, Flexor Carpi Ulnaris, Flexor Carpi Radialis, Palmaris Longus and Pronator Teres muscles. Be- fore length optimization, the model was already under $10 \%$ rRMSE in musculotendon length fitting. Depending on the accuracy requested for a given simulation, this step in the proposed method could be skipped.

As it can be seen in Table 3, a significant correlation (third quartile 0.9998) is obtained for musculotendon length while moment arm rRMSEs for WDV and WFE axis were around $40 \%$. WDV and WFE axis moment arm were ten times shorter than EFE and PS axis moment arm, so their impact was less significant on musculotendon length. This also meant that having a significant musculotendon length correlation did not imply having low moment arm rRMSE.

Muscle path resulting from this use case may be taken with caution: input data is a polynomial model [17] depicting another model [7], which was itself manually adjusted to fit experimental data. For instance, for the Extensor Carpi Ulnaris muscle, the polynomial data have a rather high error (86.63\% error for WDV moment arm). It is therefore not surprising that the method had difficulty to approximate a model that is poorly representing a muscle path. In addition, a model fitting this polynomial perfectly would be very far from [7] model, because of the initial high error rate. Using experimental data as input data should improve the realism of the generated muscle paths.

The model used in the example was a $170 \mathrm{~cm}$ tall generic male model. Generally, in a musculoskeletal simulation, it is from this musculoskeletal model that the scaling is performed to fit the specific anatomy of the subject. The interest of using a model with only via points is to go beyond the problem of scaling wrapping objects. Scaling wrapping objects is more complex since it requires to scale an object defined by multiple parameters. There is not any obvious scaling rules for these parameters, since literature often reports user intervention $[12,15]$. 

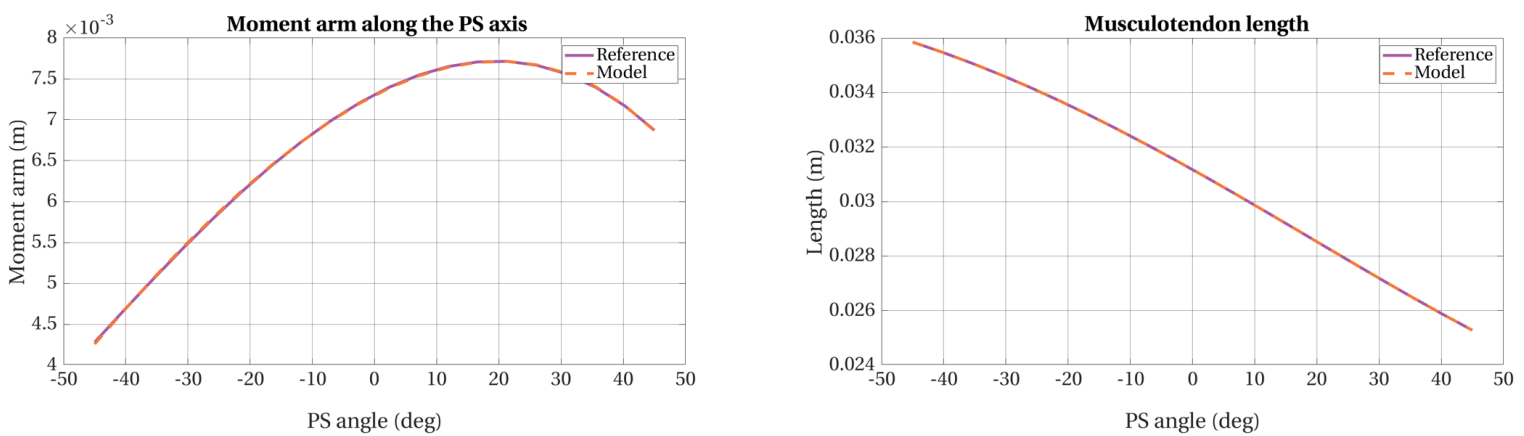

Figure 9: Example of results for Pronator Quadratus muscle. Maximum moment arm error was 22.1 $\mu$ m (0.29\% of maximum moment arm) and maximum musculotendon length error was $2.10 \mu \mathrm{m}(0.01 \%$ of maximum musculotendon length)
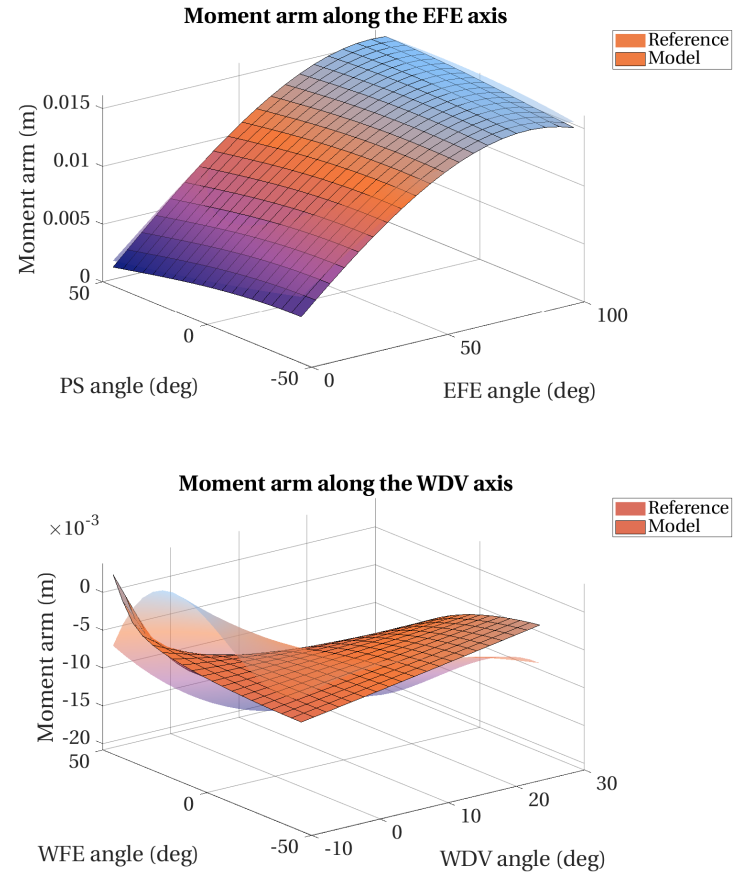
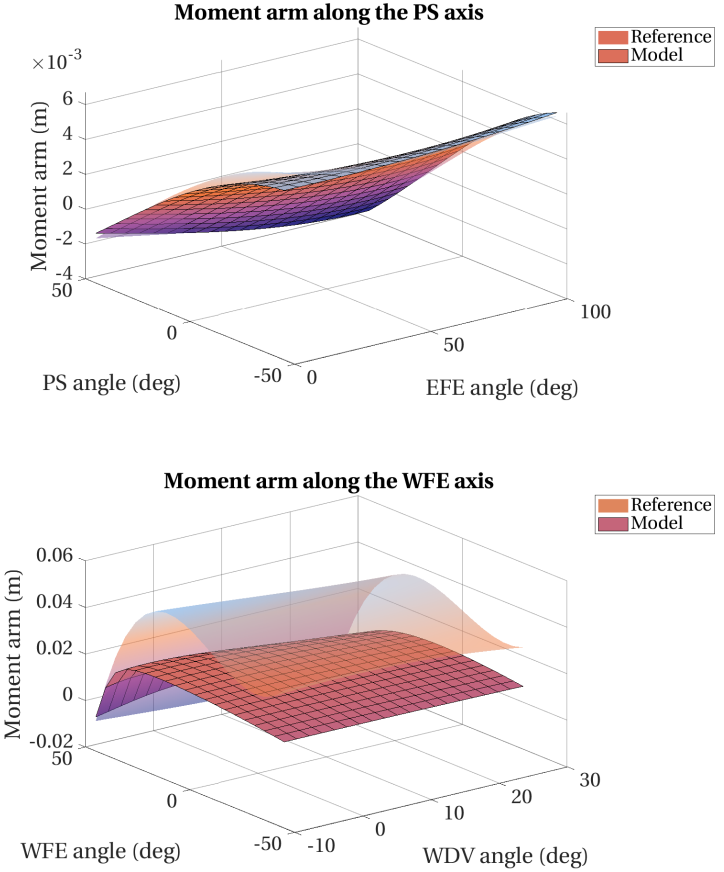

Figure 10: Example of results for Palmaris Longus muscle. Maximum error for each joint was: EFE: 1.7mm (10.69\% of maximum EFE moment arm), PS: $0.96 \mathrm{~mm}$ (14.32\% of maximum PS moment arm), WDV: $9.41 \mathrm{~mm}$ (45.13\% of maximum WDV moment arm), WFE: $29.5 \mathrm{~mm}$ (59.93\% of maximum WFE moment arm)

\subsection{Method limitations}

Computation time is gained as soon as there are more than two obstacles in the input data model (Table 2). Muscle moment arms are derived from musculotendon lengths, so they can be computed through a musculotendon length discretization. The critical computation time in moment arm computation for muscle forces estimation is therefore located in the computation time of the musculotendon length at a given angle. However, to compute a muscle path length with at least two wrapping surfaces, it is necessary to go through an optimization routine [11]. For via points, the musculotendon length is the norm between all the points of the path. Computational cost of via points is far less expensive than computational cost of obstacles [12]. The method did not provide any computational time gain for the Brachialis, which already had only two via points, as it can be seen in
Table 2. For all the other muscles with a single obstacle, the gain in terms of complexity and computation time still has to be quantified to draw a conclusion as to the usefulness of the method for them.

Nevertheless, there is a temporal gain in modeling muscle path. The method is systematic and its parameters are automatically chosen: the user only needs to place origin and insertion points and does not have to choose and adjust obstacles for each muscle path.

The optima found by the algorithm are not guaranteed to be global optima of (4). However, with regards to literature, the obtained optima are relevant enough to be used, as it can be seen in next paragraph. 


\subsection{From model to reality}

According to the Zajac-Hill model [26], muscle force is related to muscle length. The muscle force generation parameters of a musculoskeletal model, such as the forcelength relationship, can be scaled for a subject by measuring torques at a given position [27]. Such parameters can be overfitted to compensate possible errors on moment arm and musculotendon length values, affecting the estimation of muscle forces in musculoskeletal simulation. It is therefore best to properly scale these values, prior to any force generation parameters adjustment.

The model resulting from the method was realistic from a kinematic point of view: the contribution of a muscle to a joint can be realistically computed as long as experimental kinematic data are used as input data of the simulation. However, from an anatomical point of view, no muscle is composed of straight lines. This model can be sufficient as long as interactions between muscles or with other soft tissues, like friction, are not needed in a musculoskeletal simulation. The possible bone penetration was not considered and the body volumes were used only to restrict via point positions, meaning that the whole muscle path may not be contained in it.

\section{Conclusion}

In order to simplify tedious muscle modeling, this study proposed an automatic method which creates a systematic muscle path with only via points fitting moment arm and musculotendon length input.

The simplification aimed at three majors goals: avoiding scaling issues of wrapping objects; avoiding specific muscle modeling; and avoiding manual muscle path parameterization. Using only via points achieved the first goal, using a systematic parameterization achieved the second goal, and using an optimization method to automatically find the via point positions achieved the third goal.

Different methods of automatic via point placement were developed for type 1 and type 2 muscles. For type 1 muscles, the method relied on a minimization of musculotendon length while respecting spatial boundaries and moment arm input data data. For type 2 muscles, the method relied only on minimizing the distance between moment arm model and moment arm input data, while respecting spatial boundaries. Origin and insertion points were then moved according to an homothetic rule to fit the musculotendon lengths.

This method was applied to a forearm model from [16], to test its relevance. The input musculotendon length and moment arm were polynomial data from [17], while initial origin and insertion points were taken from [7].

Moment arm results lied in literature variation range: for $75 \%$ of muscles, rRMSE is under $29.23 \%$ for moment arm. EFE and PS moment arms were better approximated than WDV and WFE moment arms. Musculotendon length rRMSE were all upon $11.18 \%$. The method is particularly relevant for muscles actuating a single joint.

The main benefit of this method is its usability: each muscle path can be automatically created from its moment arm, musculotendon length, origin and insertion points data. This functionality is available in the CusToM musculoskeletal simulation library [23]. A user can simply gather the proper data, indicate the origin and insertion points to the method and let the muscle paths being automatically computed.

Further studies should investigate the pertinence of different scaling rules to apply to the via points generated using this method, as well as evaluate the computation time gained from using simplified muscles paths. The anatomical realism of the generated path remains a limitation of the proposed method.

In a near future, we plan to apply this method with experimental data for the lower limb, as those available in [28].

\section{References}

[1] Erdemir, A., McLean, S., Herzog, W., and van den Bogert, A. J., 2007. "Model-based estimation of muscle forces exerted during movements". Clinical Biomechanics, 22(2), pp. 131-154.

[2] Herrmann, A. M., and Delp, S. L., 1999. "Moment arm and force-generating capacity of the extensor carpi ulnaris after transfer to the extensor carpi radialis brevis". Journal of Hand Surgery, 24(5), pp. 1083-1090.

[3] Garner, B. A., and Pandy, M. G., 2001. "Musculoskeletal model of the upper limb based on the visible human male dataset". Computer Methods in Biomechanics and Biomedical Engineering, 4(2), pp. 93-126.

[4] Garland, A. K., Shah, D. S., and Kedgley, A. E., 2018. "Wrist tendon moment arms: Quantification by imaging and experimental techniques". Journal of Biomechanics, 68, pp. 136-140.

[5] Murray, W. M., Delp, S. L., and Buchanan, T. S., 1995. "Variation of muscle moment arms with elbow and forearm position". Journal of Biomechanics, 28(5).

[6] Gonzalez, R. V., Buchanan, T. S., and Delp, S. L., 1997. "How muscle architecture and moment arms affect wrist flexion-extension moments". Journal of Biomechanics, 30(7), pp. 705-712.

[7] Holzbaur, K. R., Murray, W. M., and Delp, S. L., 2005. "A model of the upper extremity for simulating musculoskeletal surgery and analyzing neuromuscular control". Annals of Biomedical Engineering, 33(6), pp. 829-840.

[8] Jensen, R. H., and Davy, D. T., 1975. "An investigation of muscle lines of action about the hip: A centroid line approach vs the straight line approach". Journal of Biomechanics, 8(2).

[9] Song, D., Lan, N., Loeb, G. E., and Gordon, J., 2008. "Model-based sensorimotor integration for multi-joint control: Development of a virtual arm model". Annals of Biomedical Engineering, 36(6), pp. 1033-1048.

[10] Gatti, C. J., and Hughes, R. E., 2009. "Optimization of muscle wrapping objects using simulated annealing". Annals of Biomedical Engineering, 37(7), pp. 13421347.

[11] Garner, B. A., and Pandy, M. G., 2000. “The obstacle- 
set method for representing muscle paths in musculoskeletal models". Computer Methods in Biomechanics and Biomedical Engineering, 3(1), pp. 1-30.

[12] Favre, P., Gerber, C., and Snedeker, J. G., 2010. "Automated muscle wrapping using finite element contact detection". Journal of Biomechanics, 43(10), pp. 19311940.

[13] Agarwal, P., Kuo, P. H., Neptune, R. R., and Deshpande, A. D., 2013. "A novel framework for virtual prototyping of rehabilitation exoskeletons". IEEE International Conference on Rehabilitation Robotics.

[14] Puchaud, P., Sauret, C., Muller, A., Bideau, N., Dumont, G., Pillet, H., and Pontonnier, C., 2020. "Accuracy and kinematics consistency of marker-based scaling approaches on a lower limb model: a comparative study with imagery data". Computer Methods in Biomechanics and Biomedical Engineering, 23(3), pp. 114-125.

[15] Lund, M. E., Andersen, M. S., de Zee, M., and Rasmussen, J., 2015. "Scaling of musculoskeletal models from static and dynamic trials". International Biomechanics, 2(1), pp. 1-11.

[16] Pennestrì, E., Stefanelli, R., Valentini, P. P., and Vita, L., 2007. "Virtual musculo-skeletal model for the biomechanical analysis of the upper limb". Journal of Biomechanics, 40(6), jan, pp. 1350-1361.

[17] Rankin, J. W., and Neptune, R. R., 2012. "Musculotendon lengths and moment arms for a three-dimensional upper-extremity model". Journal of Biomechanics, 45(9), pp. 1739-1744.

[18] Sherman, M. A., Seth, A., and Delp, S. L., 2013. "What is a moment arm? Calculating muscle effectiveness in biomechanical models using generalized coordinates". Proceedings of the ASME Design Engineering Technical Conference, 7 B, pp. 1-9.

[19] An, K. N., Takahashi, K., Harrigan, T. P., and Chao, E. Y., 1984. "Determination of muscle orientations and moment arms". Journal of Biomechanical Engineering, 106(3), pp. 280-282.

[20] Wu, G., Van Der Helm, F. C., Veeger, H. E., Makhsous,
M., Van Roy, P., Anglin, C., Nagels, J., Karduna, A. R., McQuade, K., Wang, X., Werner, F. W., and Buchholz, B., 2005. "ISB recommendation on definitions of joint coordinate systems of various joints for the reporting of human joint motion - Part II: Shoulder, elbow, wrist and hand". Journal of Biomechanics, 38(5), pp. 981-992.

[21] Dumas, R., Chèze, L., and Verriest, J. P., 2007. "Adjustments to McConville et al. and Young et al. body segment inertial parameters". Journal of Biomechanics, 40(3), pp. 543-553.

[22] Yeadon, M. R., 1990. "The simulation of aerial movement-II. A mathematical inertia model of the human body". Journal of Biomechanics, 23(1), pp. 67-74.

[23] Muller, A., Pontonnier, C., Puchaud, P., and Dumont, G., 2019. "CusToM: a Matlab toolbox for musculoskeletal simulation". Journal of Open Source Software, 4(33), jan, p. 927.

[24] Murray, W. M., Buchanan, T. S., and Delp, S. L., 2002. "Scaling of peak moment arms of elbow muscles with upper extremity bone dimensions". Journal of Biomechanics, 35(1), pp. 19-26.

[25] Goislard De Monsabert, B., Edwards, D., Shah, D., and Kedgley, A., 2018. "Importance of Consistent Datasets in Musculoskeletal Modelling: A Study of the Hand and Wrist". Annals of Biomedical Engineering, 46(1), pp. 71-85.

[26] Zajac, F. E., 1989. Muscle and tendon: properties, models, scaling, and application to biomechanics and motor control.

[27] Wu, W., Lee, P. V., Bryant, A. L., Galea, M., and Ackland, D. C., 2016. "Subject-specific musculoskeletal modeling in the evaluation of shoulder muscle and joint function". Journal of Biomechanics, 49(15), pp. 36263634.

[28] Buford, W. L., Ivey, F. M., Malone, J. D., Patterson, R. M., Pearce, G., Nguyen, D. K., and Stewart, A. A., 1997. "Muscle balance at the knee-moment arms for the normal knee and the acl-minus knee". IEEE Transactions on Rehabilitation Engineering, 5(4), pp. 367379. 